\title{
Interaction of CTX-M-15 enzyme with cefotaxime: a molecular modelling and docking study
}

\author{
Shazi Shakil, Asad Ullah Khan* \\ Interdisciplinary Biotechnology Unit, Aligarh Muslim University, Aligarh-202002, India, \\ Asad U. Khan - Email - asad.k@rediffmail.com, Phone: +919837021912 Fax: +91-571-2721776; *Corresponding author
}

Abstract:

Received December 15, 2009; revised February 10, 2010; accepted March 07, 2010; published April 30, 2010

Extended-spectrum $\beta$-lactamases (ESBLs) are the bacterial enzymes that make them resistant to advanced-generation cephalosporins. CTX$\mathrm{M}$ enzymes (the most prevalent ESBL-type) target cefotaxime. Aims of the study were: (i) Modelling of CTX-M enzyme from bla $a_{\mathrm{CTX}-\mathrm{M}}$ sequences of clinical Escherichia coli isolates (ii) Docking of cefotaxime with modelled CTX-M enzymes to identify amino acid residues crucial to their interaction (iii) To hypothesize a possible relationship between 'interaction energy of the docked enzyme-antibiotic complex' and 'minimum inhibitory concentration (MIC) of the antibiotic against the bacteria producing that enzyme'. Seven E. coli strains of clinical origin which were confirmed as PCR-positive for bla $a_{\mathrm{CTX}-\mathrm{M}}$ were selected for the study. C600 cells harboring cloned $b l a_{\mathrm{CTX}-\mathrm{M}}$ were tested for ESBL-production by double-disk-synergy test. BLAST analysis confirmed all the $b a_{\mathrm{CTX}-\mathrm{M}}$ genes as bla $a_{\mathrm{CTX}-\mathrm{M}-15}$. Four of the 7 strains were found to be clonally related. Modelling was performed using Swiss Model Server. Discovery Studio 2.0 (Accelrys) was used to prepare Ramachandran plots for the modelled structures. Ramachandran Z-scores for modelled CTX-M enzymes from E. coli strains D8, D183, D253, D281, D282, D295 and D296 were found to be $-0.449,0.096,0.027,0.043,0.032,-1.249$ and -1.107 , respectively. Docking was performed using Hex 5.1 and the results were further confirmed by Autodock 4.0. The amino acid residues Asn 104, Asn132, Gly 227, Thr 235, Gly 236, and Ser237 were found to be responsible for positioning cefotaxime into the active site of the CTX-M-15 enzyme. It was found that cefotaxime MICs for the CTX-M-15-producers increased with the increasing negative interaction energy of the enzyme-antibiotic complex.

Keywords: antibiotic resistance; CTX-M; docking; extended-spectrum $\beta$-lactamases; modelling.

Background:

Antibiotic resistance in bacteria is an increasing problem worldwide [1]. Extended spectrum $\beta$-lactamases (ESBLs) are the bacterial enzymes that make them resistant to advanced generation cephalosporins and might lead to therapeutic dead-end. ESBLs are of due scientific concern because they are often plasmid-associated and there can be cross-species dissemination of these plasmids. Moreover, these plasmids often carry genes for co-resistance to other antibiotics such as aminoglycosides, fluoroquinolones, tetracyclines, chloramphenicol and sulfamethoxazole-trimethoprim. ESBLs hydrolyze the $\beta$-lactam ring present in the target antibiotics. CTX-M enzymes have become the most prevalent ESBLs [2] which mainly target cefotaxime. The change in activities of CTX-Ms leading to the evolution of more variants may be due to point mutations present either inside or outside of the active site omega loop (amino acid positions 161 to 179) [3]. Identification of the amino acid residues crucial to the interaction between CTX-M (the enzyme produced by resistant bacteria) and cefotaxime (the drug hydrolyzed by this bacterial enzyme) is a problem of deep scientific interest. This information might be useful for the scientists involved in drug-designing in their search for more potent and versatile CTX$\mathrm{M}$ inhibitors. A number of authors have explored the binding of different variants of CTX-M (other than CTX-M-15, which is being covered by the present study) with reference to structure and dynamics. Kimura et al [4] have described binding of cefotaxime to CTX-M-18 and CTX-M-19. Notably, the authors observed that although the oxime side chain of cefotaxime does not have a carboxylate group to form hydrogen bond with Ser237, Ser237 kept an original conformation without a steric interaction with the methoxime in CTX-M-18. The C-4 carboxylate formed hydrogen bonds with Ser130 and Arg276. On the other hand, the steric interaction between the AT moiety and Ser167 in CTX-M-19 invoked a conformational change of Ser237 to form a hydrogen bond with the C-4 carboxylate. Thus, the C-4 carboxylate formed the hydrogen bond with Ser130 in both CTX-M-18 and CTX-M-19. The authors highlighted that their results were compatible with the fact that both enzymes have a similar affinity for cefotaxime [4]. Regarding the inhibitors, sulbactam, clavulanate and tazobactam are traditional CTX-M inhibitors, while LN1-255 and NXL-104 [5] are novel inhibitors. Most of these share the same binding site as cefotaxime. For instance, sulbactam forms a dead-end-complex with CTX-M and renders it ineffective and occupies the same site as cefotaxime. To the best of our knowledge, there are no studies reporting modelling of CTX-M-15 variant of the CTX-M family and their docking with cefotaxime. Also, there was no X-ray crystallographic structure available with the Protein Data Bank for this variant of CTX-M family at the time of communicating this paper.In view of the stated background, we found it pertinent to study the mode of interaction of CTX-M enzyme with cefotaxime. This study is based on interaction energies. The objectives of the study were: (i) Modelling of CTX-M enzyme from bla $a_{\mathrm{CTX}-\mathrm{M}}$ sequences of clinical Escherichia coli isolates (ii) Docking of cefotaxime with modelled CTX-M enzymes in order to identify amino acid residues crucial to their interaction (iii) To hypothesize a possible relationship between 'interaction energy of the docked enzyme-antibiotic complex' and 'minimum inhibitory concentration (MIC) of the antibiotic against the bacteria producing that enzyme'.

\section{Methodology:}

Seven E. coli strains of clinical origin which were confirmed as PCR-positive for $b l a_{\text {СтХ-M }}$ were selected for the present study. Originally, 111 clinical $E$. coli isolates that gave a positive doubledisk synergy test for ESBL-production were screened for the presence of bla $a_{\mathrm{CTX}-\mathrm{M}}$ gene by PCR. As these 7 strains were PCRpositive for bla $a_{\mathrm{CTX}-\mathrm{M}}$, we narrowed down to the same from amongst these strains for further investigations. Strains D8, D183, D282, D295 and D296 were isolated from neonates admitted to neonatal intensive care unit of Aligarh Hospital, India. Strains D253 and D281 were isolated from infected foot ulcers of diabetic patients admitted to the endocrinology ward of the same hospital. The bla $_{\text {CTX-M }}$ genes which were amplified from clinical E. coli strains were cloned into E. coli C600 cells using 'Qiagen cloningplus kit' as per manufacturer's instructions (Qiagen, USA). C600 blaстХ-M cells were tested for ESBL-production by the double-disk synergy test performed as described by Jarlier et al [6]. Briefly the synergy test with ceftazidime, cefotaxime, ceftriaxone and cefixime was performed by disk diffusion method on Mueller-Hinton agar plates with and without $10 \mu \mathrm{g}$ of amoxyclav. $\mathrm{A} \geq 5-\mathrm{mm}$ increase in the zone of diameter of third generation cephalosporins, tested in combination with amoxyclav versus its zone when tested alone was considered indicative of ESBL production. E. coli ATCC 25922 was used as ESBL negative and Klebsiella pneumoniae 700603 was used as ESBL positive reference strain. Also C600 blacTX-M cells were again tested for the presence of the said gene by PCR. Cefotaxime and ceftazidime MICs for the study strains were determined by the Clinical and Laboratory Standards Institute (CLSI)-microbroth dilution method. Antibiotic susceptibilities were tested by disk-diffusion method as per the CLSI guidelines for the 


\section{Bioinformation}

same. Transmissibility of cefotaxime and ceftazidime resistance markers was checked by transconjugation which was performed as described previously [7]. Enterobacterial repetitive intergenic consensus (ERIC)-PCR was used for the analysis of genetic relatedness of the seven selected strains as described by Versalovic et al [8]. BLAST-P was performed to retrieve suitable templates for homology modelling using the study $b l a_{\text {СTX-M }}$ sequences. Protein Data Bank (PDB) IDs of the templates retrieved for modelling are shown in table 1. Modelling was performed using Swiss Model Server [9]. The modelled structures (prior to docking) were verified using the "Structure Assessment" function of the Swiss Model Workspace which incorporates Procheck [10] and verify3D programmes [11]. PDB structure of cefotaxime was retrieved from Drug bank (Drug Bank accession no. DB00493). The ligand (cefotaxime) was docked into each of the modelled structures employing the Hex 5.1 software. This program uses spherical polar Fourier correlation to accelerate docking calculations. However, since Hex 5.1 is generally considered a more reliable platform for "protein-protein docking" compared to "protein-ligand" docking, we reconfirmed our results using the targeted docking mode of 'Molecular Docking Server' which is based on the well known Autodock program. The MMFF94 force field was used for energy minimization of ligand molecules. Gasteiger partial charges were added to the ligand atoms. Non-polar hydrogen atoms were merged, and rotatable bonds were defined. Docking calculations were carried out on the protein models. Essential hydrogen atoms, Kollman united atom type charges, and solvation parameters were added with the aid of AutoDock tools. Affinity (grid) maps of $60 \times 60 \times 60 \AA$ grid points (centred on Ser237, a well identified residue of the active site) and $0.375 \AA$ spacing were generated using the Autogrid program. AutoDock parameter set and distancedependent dielectric functions were used in the calculation of the van der Waals and the electrostatic terms, respectively. Docking simulations were performed using the 'Lamarckian genetic algorithm (LGA)' and the 'Solis \& Wets local search method'. Each docking experiment was derived from 10 different runs that were set to terminate after a maximum of 250000 energy evaluations. The population size was set to 150 . During the search, a translational step of $0.2 \AA$, and quaternion and torsion steps of 5 were applied. The lowest-energy structures were selected as energy-refined complex models. The models were duly verified by Procheck [10] and verify3D programmes [11]. Discovery Studio 2.0 (Accelrys) was used to prepare Ramachandran plots for the modelled structures. Ramachandran Z-scores for the evaluation of these models were calculated using 'WHAT IF' package. Alignments were performed by ClustalW program using default parameters.

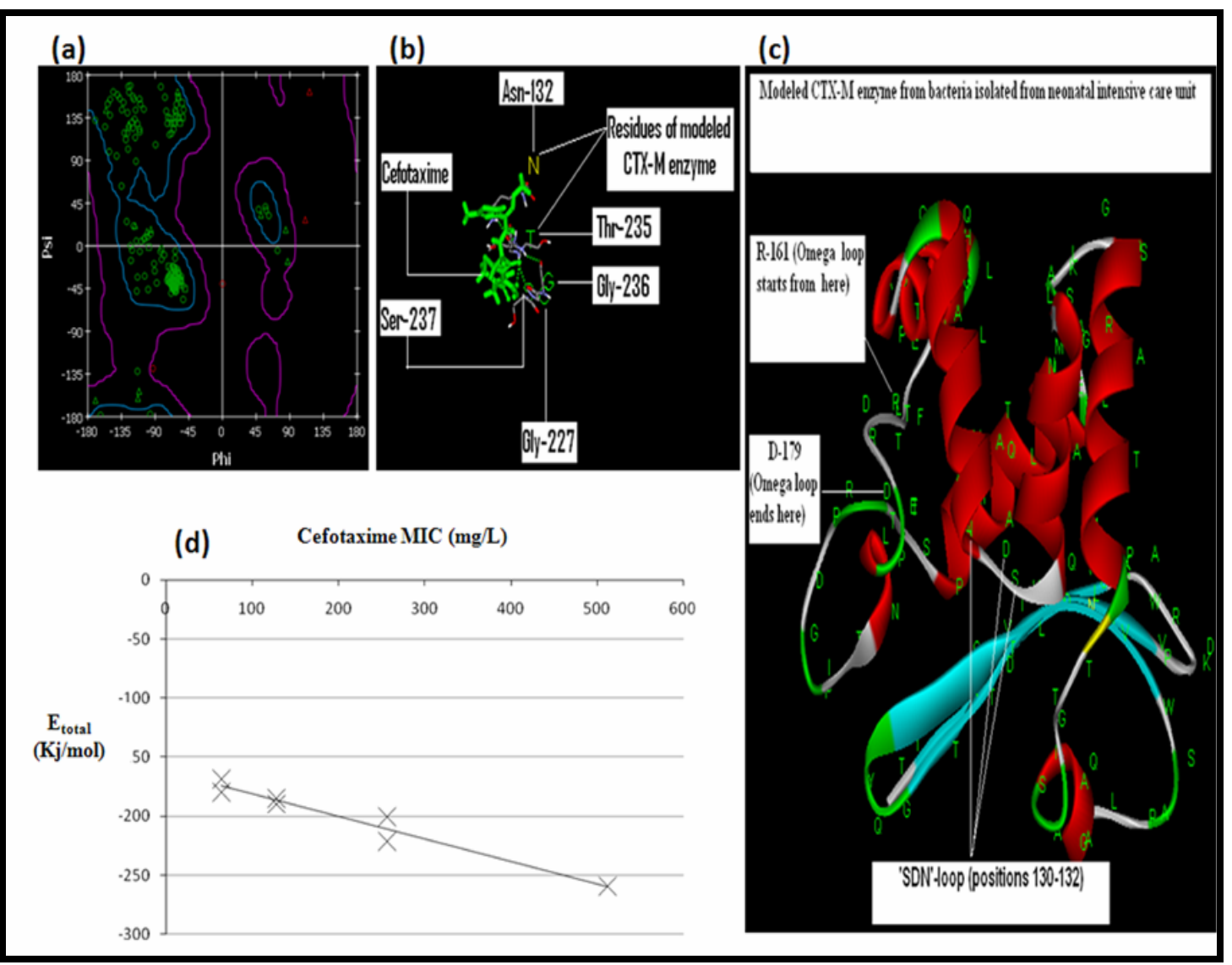

Figure 1 (a) Ramachandran plot for the modelled CTX-M-15 enzyme corresponding to strain D8 (GenBank accession no. - FJ997864) (b) Interaction between cefotaxime and the modelled CTX-M-15 enzyme obtained by Hex5.1 (c) Modelled CTX-M-15 enzyme corresponding to strain D8 (d) Total interaction energy $\left(\mathrm{E}_{\text {total }}\right)$ versus cefotaxime MIC graph for the study E. coli strains (Docking: Ligand = cefotaxime; Receptor $=$ modelled CTX-M-15 enzymes). 


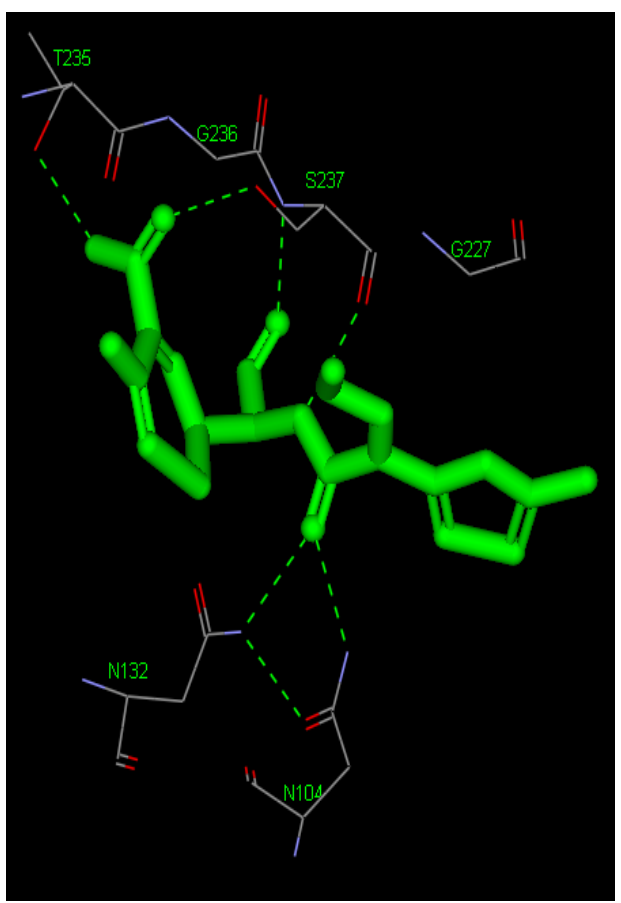

Figure 2 Interaction between cefotaxime and the modelled CTX-M-15 enzyme obtained using Autodock4.0. The drug is shown in stick form. The broken lines represent hydrogen bonds. 'Interacting amino acid residues' are labeled. Figure has been produced by Discovery Studio 2.0

\section{Discussion:}

BLAST analysis of the sequences confirmed all the $b l a_{\mathrm{CTX}-\mathrm{M}}$ genes as $b a_{\mathrm{CTX}-\mathrm{M}-15}$. The gene sequences used in the study appear in GenBank with the following accession numbers: FJ997864, FJ997865， FJ997866， GQ145220， GQ145221， GQ174503 and GQ174504. The cefotaxime resistance markers were found to be located on the plasmid DNA of the study strains and were transmissible by conjugation. Conjugation frequencies were of the order $10^{-5}$. Four out of the 7 study strains were found to be clonally related as revealed by their ERIC-profiles. The bla $a_{\mathrm{CTX}-\mathrm{M}-15}$ from strain D8 possessed point mutations $262 \mathrm{C} \rightarrow \mathrm{A}$ and $265 \mathrm{~A} \rightarrow \mathrm{G}$ resulting in amino acid substitutions Pro88Thr and Asn89Asp respectively (Table 1). In the strain D295, bla $a_{\mathrm{CTX}-\mathrm{M}-15}$ possessed point mutation $689 \mathrm{G} \rightarrow \mathrm{C}$ leading to the amino acid substitution Val230Leu. Additional information regarding sequence-alignments and secondary structures is presented in supplementary files 1 and 2. Over $90 \%$ of the amino acid residues in the protein structures modelled from $b l a_{\text {СтХ-М-15 }}$ genes were found to be present in the most favored regions as revealed by their respective Ramachandran plots. For instance, percent amino acid residues in most favored, additional allowed, generously allowed and disallowed regions of the Ramachandran plot for the modelled CTX-M enzyme corresponding to strain D8 (GenBank accession no.- FJ997864) were $91.0 \%, 8.2 \%, 0.8 \%$ and $0.0 \%$, respectively (Figure 1a). All the enzyme structures were modelled using the PDB structure liys A as template. The target sequences possessed more than $80 \%$ sequence-identity with the said template. For instance, D8 CTX-M

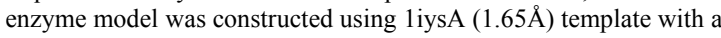
sequence-identity of $85.326 \%$ (E value: $5.27 \mathrm{e}-83$ ). Ramachandran Z-score expresses how well the backbone conformations of all the residues correspond to the known allowed areas in the Ramachandran plot. Accordingly, the Ramachandran Z-scores for modelled CTX-M enzymes from E. coli strains D8, D183, D253, D281, D282, D295 and D296 were found to be $-0.449,0.096,0.027$, $0.043,0.032,-1.249$ and -1.107 , respectively. This further confirms the accuracy of the modelled structures. Cefotaxime was docked into each of these modelled enzyme-structures.

The flexibility of the $\beta 3$ strand, omega loop, and residues Ser-237, Asp-240, and Arg-276 are known to be involved in the ISSN 0973-2063 (online) 0973-8894 (print) Bioinformation 4(10):468-472 (2010) cefotaximase activity of CTX-M enzymes [12]. It is reported that the $\beta 3$ strand of CTX-M enzymes has numerous Gly residues and is therefore probably more flexible than that of TEM enzymes. Residue Gly-240 in CTX-M-15 may further increase the flexibility of the $\beta 3$ strand and alter its positioning during the catalytic process. It is supposed that CTX-M-15 might have evolved from CTX-M-3 due to a single amino acid change (D240G). As a result of this substitution (D240G), interaction between the residues Gly 240 and Asn 270 was found to be absent in the CTX-M-15 enzyme. The absence of this interaction in CTX-M-15 might increase the flexibility of the C-terminal of the $\beta 3$ strand. During the catalytic process, this flexibility could favour the accommodation of more varied substrates with respect to their size and 3D-structures. This explanation is compatible with the fact that the CTX-M-15 enzyme is known to hydrolyze cefotaxime, ceftazidime as well as cefepime antibiotics quite efficiently. Moreover, these results are in coherence with the observation of several authors with reference to other CTXM-variants such as CTX-M-27 [13]. The SDN (positions 130-132), and KTG (positions 234-236) sequences, were found to be conserved in all the CTX-M-15 sequences obtained in this study. These are typical structure of class A enzymes [14]. All the sequences harbored the $\mathrm{D} 240 \mathrm{G}$ mutation with respect to CTX-M-3, the distinguishing feature of CTX-M-15 enzymes. None of the mutations obtained in our CTX-M-15 sequences lied within the omega loop or other positions that are known to affect the substrate profile of these enzymes. Hence the trend in the MIC values for cefotaxime and ceftazidime antibiotics in these isolates was typical of CTX-M-15 producers (Table 1). The study isolates displayed cefotaxime MICs up to 8 fold higher than ceftazidime MICs, as observed previously [15]. Figure $\mathbf{1 b}$ and figure 2 show close-up view of interaction between cefotaxime and CTX-M-15 enzyme modelled from $b l a_{\text {СтХ-M-15 }}$ gene sequence of strain D8, as per the results of Hex5.1 and Autodock4.0, respectively. It is important to mention here that the docking results obtained by Hex 5.1 were in harmony with the results obtained using Autodock 4.0 but were not identical. All, the results with reference to energies and interacting residues were same, except that Autodock identified Asn104 also as one of the interacting residues. Analysis of the docked structures by Discovery Studio2.0 was performed by using the "show ligand interactions" function of the "scripts" menu provided within the said 
software. It was revealed that the amino acid residues Asn 104, Asn132, Gly 227, Thr 235, Gly 236, and Ser237 were responsible for the proper positioning of cefotaxime into the active site of the CTX-M-15 enzyme, as per the Autodock results (which is generally considered to be more reliable than Hex5.1). This finding is supported by the views of other authors as well [16, 17]. Ramachandran Z-scores obtained by 'WHATIF' for all the docked structures were well inside tolerable limits. For instance, Z-score of the docked complex involving CTX-M enzyme model (corresponding to the strain D183) and cefotaxime, was found to be 0.390. Furthermore, the residues involved in the interaction constituted the same secondary structures as those involved in interaction of cefotaxime to closely related enzymes whose X-ray crystallographic structures are available with the 'RCSB Protein Data Bank'. Asn 132, one of the interacting residues forms part of a 'loop' in our study. Similarly, several crystallographic structures of Class A beta lactamases complexed with ligands, show Asn 132 as an interacting residue which constitutes a 'loop'. Our results (secondary structures constituted by the interacting residues as well as the docking pose) are in perfect harmony with the PDB entry 1IYO, which shows Toho-1 (a Class A beta-lactamase) in complex with cefotaxime. The first and last residues of the omega loop are clearly marked in Fig 1c, which are Arg161 and Asp179, respectively. Asn132, one of the residues important for positioning the drug into the active site of the enzyme is also shown in the figure as part of the 'SDN' loop (Figure 1c). Ser237 forms a hydrogen bond with the carboxylate oxygen of cefotaxime (Figure. 2). This interaction might induce the rotation of the carboxylate group in the acyl-intermediate structure of CTX-M-15 compared with those of non-ESBL enzymes. Interaction between Ser 237 and cefotaxime probably helps to bring the beta lactam carbonyl group to the suitable position in the oxyanion hole, thereby promoting drug-hydrolysis. The acylamide side chain of cefotaxime interacts with the $\mathrm{N}^{\delta}$ of Asn132 and the backbone oxygen of Ser 237. The $\mathrm{O}^{\delta}$ of Asn104 interacts with the $\mathrm{N}^{\delta}$ of Asn132 in the docked complex. Furthermore, $\mathrm{N}^{\delta}$ of Asn104 was found to make a hydrogen bond with the carbonyl oxygen of the acylamide side chain of cefotaxime (Figure 2). In this way, we found that a total of 7 hydrogen bonds are crucial for the correct positioning of cefotaxime within the active site of CTX-M-15 enzyme.A noteworthy relationship between total interaction energy $\left(\mathrm{E}_{\text {total }}\right)$ and cefotaxime (MICs) of the bla $_{\mathrm{CTX}-\mathrm{M}-15}$ positive study strains was observed. Cefotaxime MICs increased with the increasing negative value of interaction energy, when the former was docked into the modelled CTX-M-15 structures of the respective strains (Figure 1d). It can be explained by the fact that higher negative interaction energy for the enzymeantibiotic complex is an indicator of more stable and effective interaction between the two during binding. A more stable and better fitting of the $\beta$-lactam antibiotic (cefotaxime) into the active site of the CTX-M-15 enzyme would ensure an easy hydrolysis of the drug. This is in coherence with one of our earlier observations in which we performed docking of the enzyme Smel with different carbapenems to compare their effectiveness against Sme1 producing bacteria [18]. It was observed that the imipenem-Sme1 complex was far more stable than the complex involving doripenem. This suggested an easier hydrolysis of imipenem by Sme- 1 and a poor hydrolysis of doripenem. This was strongly supported by the experimental studies that suggested a better activity of doripenem over other carbapenems against carbapenemase producing bacteria [19]. Moreover, in a similar 2009 study authors have used interaction energies of docking to compare the efficacy of different neuraminidase inhibitors against newly evolved strains of $\mathrm{H} 1 \mathrm{~N} 1$ viruses [20]. It is important to mention here that more experimental studies are needed to establish a precise relationship between MIC and interaction energy. We admit the limitation of the present study that we could not narrow down to a possible "energy-concentration pair" that produced effective interaction between the discussed enzyme and substrate.

\section{Conclusion:}

To the best of our knowledge we are the first to report results of docking between CTX-M-15 (receptor) and cefotaxime (ligand). Moreover, this is the first study to hypothesize a possible relationship between 'interaction energy of the docked enzymeantibiotic complex' and 'minimum inhibitory concentration (MIC) of the antibiotic against the bacteria producing that enzyme'. In conclusion, the present study reveals that the amino acid residues Asn 104, Asn132, Gly 227, Thr 235, Gly 236, and Ser237 are responsible for positioning cefotaxime into the active site of the CTX-M-15 enzyme. A total of 7 hydrogen bonds are crucial for the correct positioning of cefotaxime within the active site of CTX-M15 enzyme. Cefotaxime MICs for the CTX-M-15-producers increased with the increasing negative interaction energy for the enzyme-antibiotic complex. Researchers are expected to duly utilize this information for designing more potent and versatile CTX-Minhibitors as well as new drugs resistant to hydrolysis by CTX-M enzymes. Scope is still left to determine the 3D structures of the rapidly emerging variants of CTX-M by X-ray crystallography.

\section{Acknowledgements:}

Authors acknowledge the facilities of Distributed Information Subcentre, Interdisciplinary Biotechnology Unit, AMU, Aligarh, India. $\mathrm{SS}$ is recipient of Senior Research Fellowship from Department of Biotechnology, Govt. of India.

\section{References:}

[1] S Shakil et al, J Biomed Sci (2008) 15: 5 [PMID: 17657587]

[2] R Canton \& T M. Coque, Curr Opin Microbiol, (2006) 9: 466 [PMID: 16942899]

[3] A Baraniak et al, J Antimicrob Chemother (2002) 50: 393 [PMID: 12205064]

[4] S Kimura et al, Antimicrob Agents Chemother (2004) 48: 1454 [PMID: 15105092]

[5] T Stachyra et a, J Antimicrob Chemother (2009) 64: 326 [PMID: 19493866]

[6] V Jarlier et al, Rev Infect Dis (1988) 10: 867 [PMID: 3263690]

[7] K J Gray et al, J Antimicrob Chemother (2006) 57: 661 [PMID: 16537341]

[8] J Versalovic et al, Nucleic Acids Res (1991) 19: 6823 [PMID: 1762913]

[9] K Arnold et al, Bioinformatics (2006) 22: 195 [PMID: 16301204]

[10] R A. Laskowski et al, Nucleic Acids Res (2005) 33: D266 [PMID: 15608193]

[11] D R. Eisenberg et al, Methods Enzymol (1997) 277: 396 [PMID: 9379925]

[12] R. Bonnet et al, J Antimicrob Chemother (2003) 52: 29 [PMID: 12775683]

[13] T Shimamura et al, J Biol Chem (2002) 277: 46601 [PMID: 12221102]

[14] Z Xiong et al, Int J Antimicrob Agents (2004) 23: 262 [PMID: 15164967]

[15] S Mushtaq et al, J Antimicrob Chemother (2003) 52: 528 [PMID: 12888591]

[16] R. Bonnet, Antmicrob Agents Chemother, (2004) 48: 1 [PMID: 14693512]

[17] I. Schneider et al, Antimicrob Agents Chemother (2009) 53: 4518 [PMID: 19620330]

[18] S Shakil et al, J Chemother (2009) 21: 482 [PMID: 19933038]

[19] S Marti et al, Int J Antimicrob Agents (2009) 33: 181 [PMID: 18977642]

[20] A U Khan et al, Indian J Microbiol (2009) 49: 370 [DOI: 10.1007/s12088-009-0065-2]

Edited by N. Srinivasan

Citation: Shakil \& Khan, Bioinformation 4(10): 468-472 (2010) License statement: This is an open-access article, which permits unrestricted use, distribution, and reproduction in any medium, for noncommercial purposes, provided the original author and source are credited. 
Bioinformation

www.bioinformation.net

\section{Supplementary material:}

Table 1 Characteristics of gene sequences, modelled enzymes and enzyme-drug complexes along with cefotaxime and ceftazidime minimum inhibitory concentration (MIC)-values for E. coli strains. *Not found/Not calculated by software.

\begin{tabular}{|c|c|c|c|c|c|c|c|c|c|c|c|}
\hline \multirow[t]{2}{*}{$\begin{array}{l}\text { Strain } \\
\text { No. }\end{array}$} & \multirow{2}{*}{$\begin{array}{l}\text { Accession no. of } \\
\text { reference strain used for } \\
\text { alignment }\end{array}$} & \multirow{2}{*}{$\begin{array}{l}\text { Point } \\
\text { mutation } \\
\text { (s) }\end{array}$} & \multirow{2}{*}{$\begin{array}{l}\text { Amino acid } \\
\text { substitution } \\
\text { (s) }\end{array}$} & \multirow{2}{*}{$\begin{array}{l}\text { Substitution(s) present in secondary } \\
\text { structure (Hydrophobicity/ Ribbon } \\
\text { size/ pKa) }\end{array}$} & \multirow{2}{*}{$\begin{array}{l}\text { PDB ID of the } \\
\text { temp-late retrie- } \\
\text { ved for mod-elling }\end{array}$} & \multicolumn{3}{|c|}{$\begin{array}{l}\text { Interaction energy with } \\
\text { cefotaxime } \quad(\mathrm{kJ} / \mathrm{mol})\end{array}$} & \multicolumn{2}{|c|}{$\mathrm{MIC}(\mathrm{mg} / \mathrm{L})$} & \multirow{2}{*}{$\begin{array}{l}\text { Genbank Accession } \\
\text { no. for genes (This } \\
\text { study) }\end{array}$} \\
\hline & & & & & & E-total & E-shape & E-force & $\begin{array}{l}\text { Cefo- } \\
\text { taxime }\end{array}$ & $\begin{array}{l}\text { Ceft- } \\
\text { azidime }\end{array}$ & \\
\hline D8 & EU979558.1 & $\begin{array}{l}262 \mathrm{C} \rightarrow \mathrm{A}, \\
265 \mathrm{~A} \rightarrow \mathrm{G}\end{array}$ & $\begin{array}{l}\text { Pro88Thr, } \\
\text { Asn89Asp }\end{array}$ & $\begin{array}{l}\text { Turn }(-0.7 / 0.225 / *) \text {, Turn } \\
(-3.5 / 0.225 / 3.9)\end{array}$ & 1iysA & -184.6 & -163.6 & -21 & 128 & 64 & FJ997864 \\
\hline D183 & EU979558.1 & * & * & * & 1iysA & -189.49 & -181.05 & -8.44 & 128 & 64 & FJ997865 \\
\hline D253 & EU979558.1 & * & * & * & 1iysA & -221.51 & -208 & -13.51 & 256 & 32 & FJ997866 \\
\hline D281 & EU979558.1 & * & * & * & 1iys A & -168.17 & -147.5 & -20.67 & 64 & 32 & GQ145220 \\
\hline D282 & EU979558.1 & $*$ & $*$ & $*$ & 1iys A & -179.24 & -150.42 & -28.82 & 64 & 64 & GQ174503 \\
\hline D295 & EU979558.1 & $689 \mathrm{G} \rightarrow \mathrm{C}$ & Val230Leu & Sheet $(3.8 / 1.3 / *)$ & 1iysA & -259.37 & -243.46 & -15.91 & 512 & 64 & GQ145221 \\
\hline D296 & EU979558.1 & $*$ & * & * & liys A & -200.32 & -191.39 & -8.93 & 256 & 32 & GQ174504 \\
\hline
\end{tabular}

